\title{
Altered Intrinsic Neuronal Interactions in the Visual Cortex of the Blind
}

\author{
David J. Hawellek, ${ }^{1,2}$ Inga M. Schepers, ${ }^{1,3}$ Brigitte Roeder, ${ }^{4}$ Andreas K. Engel, ${ }^{1}$ Markus Siegel, ${ }^{1,5,6 *}$ and Joerg F. Hipp ${ }^{1,5,6 *}$ \\ ${ }^{1}$ Department of Neurophysiology and Pathophysiology, University Medical Center Hamburg-Eppendorf, 20246 Hamburg, Germany, ${ }^{2}$ Center for Neural \\ Science, New York University, New York, New York 10003, ${ }^{3}$ Department of Neurobiology and Anatomy, University of Texas Health Science Center, \\ Houston, Texas 77030, ${ }^{4}$ Biological Psychology and Neuropsychology, University of Hamburg, 20146 Hamburg, Germany, and ${ }^{5}$ Centre for Integrative \\ Neuroscience and ${ }^{6}$ MEG Center, University of Tübingen, 72076 Tübingen, Germany
}

In congenital blindness, the brain develops under severe sensory deprivation and undergoes remarkable plastic changes in both structure and function. Visually deprived occipital cortical regions are histologically and morphologically altered and exhibit a strikingly remodeled functional state: absolute levels of neural activity are heightened and are modulated by nonvisual sensory stimulation as well as higher cognitive processes. However, the neuronal mechanisms that underlie this altered functional state remain largely unknown. Here, we show that the visual cortex of the congenitally blind exhibits a characteristic gain in frequency-specific intrinsic neuronal interactions. We studied oscillatory activity in 11 congenitally blind humans and matched sighted control subjects with magnetoencephalography at rest. We found increased spontaneous correlations of delta band $(1-3 \mathrm{~Hz})$ and gamma band $(76-128 \mathrm{~Hz})$ oscillations across the visual cortex of the blind that were functionally coupled. Local delta phase modulated gamma amplitude. Furthermore, classical resting rhythms $(8-20 \mathrm{~Hz})$ were reduced in amplitude but showed no altered correlation pattern. Our results suggest that both decreased inhibition and circuit mechanisms that support active processing are intrinsic features underlying the altered functional state of the visual cortex in congenitally blind individuals.

\section{Introduction}

A fine-tuned interplay between developmental programs and neuronal activity shapes the connectivity and function of visual cortical circuits during postnatal periods (Katz and Shatz, 1996). In congenital blindness, normal sensory experience is absent during development, and, as a result, brain areas that mainly subserve visual functions in sighted individuals undergo dramatic plastic changes in blind individuals. The visual cortex of blind individuals is smaller in terms of surface area and volume (Noppeney et al., 2005; Bridge et al., 2009; Park et al., 2009), but is increased in thickness (Bridge et al., 2009; Jiang et al., 2009; Park et al., 2009), which is consistent with disturbances in synaptic pruning and the elimination of exuberant neonatal connectivity that would normally establish the specificity of visual cortical organization based on sensory experience (Singer and Tretter,

Received April 17, 2013; revised Aug. 26, 2013; accepted Sept. 20, 2013.

Author contributions: D.J.H., B.R., A.K.E., M.S., and J.F.H. designed research; D.J.H. and I.M.S. performed research; D.J.H. analyzed data; D.J.H., M.S., and J.F.H. wrote the paper.

This work was supported by European Union Grants IST-2005-027268, NEST-PATH-043457, HEALTH-F2-2008200728, and ERC-2010-AdG-269716; German Research Foundation Grant GRK 1247/1-2, SFB 936/B2; and German Federal Ministry of Education and Research Grant 01GW0561, Neuroimage Nord. We thank Anna Best and Julia Föcker for their help in the recruitment of participants, and Till Schneider for help in data acquisition.

${ }^{*}$ M.S. and J.F.H. contributed equally to this work.

The authors declare no competing financial interests.

Correspondence should be addressed to either of the following: David J. Hawellek, Center for Neural Science, New York University, New York, NY 10003, E-mail: d.hawellek@nyu.edu; or Joerg F. Hipp, Centre for Integrative Neuroscience, University of Tübingen, 72076 Tübingen, Germany, E-mail: joerg.hipp@cin.uni-tuebingen.de.

DOI:10.1523/JNEUROSCI.1625-13.2013

Copyright $\odot 2013$ the authors $\quad 0270-6474 / 13 / 3317072-09 \$ 15.00 / 0$
1976; Movshon and Van Sluyters, 1981; Huttenlocher et al., 1982; Callaway and Katz, 1991). Strikingly, a wealth of studies has consistently demonstrated an altered functional role for occipital areas of blind individuals. The metabolic demand of the occipital cortical areas of blind humans is elevated compared with sighted humans (Wanet-Defalque et al., 1988; Veraart et al., 1990; De Volder et al., 1997). Moreover, the neural activity levels that are present in these regions are modulated by other sensory modalities and are proposed to contribute to higher cognitive capacities such as language and episodic memory (Sadato et al., 1996; Cohen et al., 1997; Büchel et al., 1998; Röder et al., 1999, 2002; Bavelier and Neville, 2002; Amedi et al., 2003, 2004; Burton, 2003; Gougoux et al., 2005; Raz et al., 2005; Collignon et al., 2009, 2011; Merabet and Pascual-Leone, 2010; Bedny et al., 2011; Schepers et al., 2012).

Together, visual cortical regions of the blind are structurally altered and may take part in nonvisual functions. These observations suggest that oscillatory neuronal interactions in visual cortex may also exhibit altered patterns, reflecting the changes in connectivity, biophysical properties, and function due to the atypical developmental process. However, the characteristics of the neuronal activity underlying the altered state of visual areas in blind individuals are still largely unknown. To address this question, we compared magnetoencephalography (MEG) data of congenitally blind adults and matched sighted control subjects recorded at rest. The task-free nature of the resting data allowed for studying the neuronal dynamics that emerged intrinsically within the altered structural and functional architecture of visual areas in blind individuals without imposing specific task con- 
straints (Raichle, 2010). To capture the structure of neuronal interactions of the visual cortex in blind and sighted individuals, we quantified two sensitive measures of neuronal coupling: cofluctuations in frequency-specific oscillatory power (Bruns et al., 2000; Leopold et al., 2003; Brookes et al., 2012; Hipp et al., 2012; Siegel et al., 2012) and cross-frequency phase to amplitude coupling (Bragin et al., 1995; Schack et al., 2002; Lakatos et al., 2005; Canolty and Knight, 2010).

\section{Materials and Methods}

Participants. Eleven congenitally blind participants ( 6 female; mean \pm SD age, $35.5 \pm 9.5$ years; age range, $23-48$ years) and 11 control subjects matched in age, gender, handedness, and education (mean \pm SD age, $36.5 \pm 10.7$ years; age range, $22-54$ years) participated in this study and received monetary compensation for their participation. The causes of blindness included retinopathy of prematurity (six participants), genetic defects (two participants), oxygen deficiency at birth (one participant), a distorted optic nerve (one participant), and retinoblastoma (one participant). Four of the congenitally blind participants had very weak residual light perception. No participant had a further history of neurological or psychiatric illness. The blind and sighted participants were blindfolded during the recording period, and all sighted participants were instructed to additionally close their eyes. Approval of the local ethics committee for this study was obtained, and the study was conducted in accordance with the Declaration of Helsinki. Informed consent was obtained from all participants before the recordings.

Data acquisition and preprocessing. MEG (275 channel; Omega 2000, CTF Systems Inc.) was recorded continuously for 5 min during silent wakefulness before the start of another experiment (Schepers et al., 2012). The electrooculogram and electrocardiogram were recorded along with the MEG. The head position relative to the MEG sensors was measured continuously using a set of head localization coils (nasion, and left and right ears). All analyses were performed in Matlab (MathWorks) using custom scripts and the open source toolboxes Fieldtrip (http://www.ru.nl/fcdonders/fieldtrip; Oostenveld et al., 2011) and SPM8 (http://www.fil.ion.ucl.ac.uk/spm). The MEG signals were lowpass filtered on-line (cutoff: $300 \mathrm{~Hz}$ ) and sampled with $1200 \mathrm{~Hz}$. Off-line, the data were split into two datasets for each participant to adaptively suppress the noise inherent to lower-frequency and higher-frequency ranges. One dataset was high-pass filtered with a cutoff frequency of 0.5 $\mathrm{Hz}$ (low-frequency dataset, fourth-order Butterworth filter), while the other dataset was high-pass filtered with a cutoff frequency of $30 \mathrm{~Hz}$ (high-frequency dataset, fourth-order Butterworth filter). Line noise was removed from both datasets by applying band-stop filters centered on 50 , 100,150 , and $200 \mathrm{~Hz}$ with cutoffs at $\pm 1 \mathrm{~Hz}$ (fourth-order Butterworth filters). Both datasets were resampled to $600 \mathrm{~Hz}$. The datasets were manually screened for artifactual periods that were excluded for further analyses. Furthermore, we used independent component analysis (FAST ICA; Hyvärinen, 1999) to remove the remaining artifactual signals. The selection of artifactual components was based on visual inspection. A component was only treated as an artifact (e.g., eye, muscle, or heart related) when the conjunction of the topography, power spectrum, and time course was clearly conclusive. Artifactual components were subtracted from the data before further analysis. The number of components that were subtracted did not differ between the groups (low-frequency dataset: Wilcoxon rank-sum tests, $p=0.57$; median number of components rejected, $n=7$; maximal number, $n=23$; minimal number, $n=3$; high-frequency dataset: Wilcoxon rank-sum tests, $p=1$; median number of components rejected, $n=8.5$; maximal number, $n=19$, minimal number, $n=3$ ).

Spectral Analyses. All spectral analyses were performed using Morlet's wavelets $w(t, f)$, as follows:

$$
\begin{gathered}
w(t, f)=\left(\sigma_{t} \sqrt{\pi}\right)^{-1 / 2} e^{-t^{2} / 2 \sigma_{t}^{2}} e^{-i 2 \pi f t} \\
X(t, f)=x(t) * w(t, f),
\end{gathered}
$$

where $f$ is the center frequency, $\sigma_{t}$ is the temporal SD, and $X(t, f)$ is the spectral estimate of the MEG signal $x(t)$. We chose a logarithmic frequency spacing of center frequencies according to the exponentiation of the base 2 with coefficients ranging from -0.25 in steps of 0.25 to 7.25 , and a bandwidth of one-half octaves (this corresponds to $\frac{f}{\sigma_{f}} \sim 5.83$, with $\sigma_{f}$ being the spectral SD). Signal power was estimated for successive half-overlapping time windows covering $\pm 3 \sigma_{t}$. For cross-frequency analysis, we chose a spectral band-width of $3 / 2$ octaves for the phase signals and 3/4 octaves for the amplitude signals as well as a slidingwindow step size of 5 samples (i.e., $\sim 8.3 \mathrm{~ms}$ ). For power and powercorrelation analyses $>46 \mathrm{~Hz}$ ( 2 to the power of 5.5), we used the dataset that was specifically preprocessed to remove high-frequency artifacts (see above).

Source localization. Frequency-specific measures were calculated after source projecting the frequency-decomposed MEG data with adaptive linear spatial filters (Van Veen et al., 1997; Gross et al., 2001) to predefined source locations. Individual head models were constructed by applying an affine transform (based on individual T1 MRI scans) to source locations (a regular grid of $1 \mathrm{~cm}$ spacing in MNI coordinates; 2925 brain source locations), bringing them into individual head space and aligning the MEG sensors to the head geometry based on three fiducial points (nasion, left and right ear, defined in the MEG by the three head localization coils). The physical relation between the sources and sensors (leadfield) was then derived using a single-shell model (Nolte, 2003). Visual regions of interest were defined as all source locations of the grid that were labeled as the according Brodmann area 17 (BA17) or BA18 in Talairach space (using mni2tal; http://imaging.mrc-cbu.cam.ac.uk/ imaging/MniTalairach). The implementation details of the frequencyspecific beamformers were as follows: for each source location, three orthogonal filters $\left[\hat{A}=\left(A_{x} A_{y} A_{z}\right)\right.$, one for each spatial dimension] were computed that pass activity from a given location with unit gain, while maximally suppressing activity from all other sources, as follows:

$$
\hat{A}(r, f)=\left[L^{T}(r) C_{\text {real }}(f)^{-1} L(r)\right]^{-1} L^{T}(r) C_{\text {real }}(f)^{-1} .
$$

Here, $L$ is a matrix whose columns are the leadfields of three orthogonal dipoles at a given source location $r$, and $C_{\text {real }}$ denotes the real part of the complex cross-spectral density matrix for the data at frequency $f$ and $T$ indicates the matrix transpose. We linearly combined the three filters to a single filter pointing in the direction of maximal variance (i.e., the dominant dipole orientation). To this end, the filters were weighted with the first eigenvectorelements (the eigenvector with the largest eigenvalue of the real part of the cross-spectral density matrix at the source location). To project the sensor data to source level, the data were then multiplied with the filter, as follows:

$$
X_{\text {source }}(r, t, f)=A X_{\text {sensor }} \text {. }
$$

The adaptive nature of the beamformer described above does not allow for deriving an unbiased estimate of absolute power. Thus, we estimated the absolute power within visual cortex (see Fig. $4 B$ ) with a nonadaptive spatial filter that passes activity from a given source location with unit gain according to the leadfield of the location, as follows:

$$
\hat{A}(r)=\frac{L(r)}{\sum L(r)^{2}} .
$$

For displaying results, we projected the data onto the inflated cortical surface of the Population-Average, Landmark and Surface-Based Atlas (Van Essen, 2005).

Power correlations. We investigated differences in brain-wide bandlimited power correlations between the blind and sighted individuals across a large range of frequencies. For statistical testing, we used a twostep procedure based on permutation statistics.

In the first step, for each frequency, we determined whether the brainwide correlation differed between the groups averaged across all brainwide correlations. To this end, we constructed correlation matrices for each subject and each frequency that contained all pairwise power correlations among the 2925 source locations of the brain-wide grid. Importantly, before computing the correlation between power time courses, we used an orthogonalization procedure, which ensured that no trivial signal covariation due to the limited spatial resolution of MEG confounded 
the estimate of power correlations (Hipp et al., 2012). Each correlation was computed twice, with the signals mutually orthogonalized in both directions, and these correlation values were averaged before further analysis. Our analyses were based on the correlations of either the visual cortex (defined as source locations in BA17 or BA18, for a total of 113 source locations) with the global grid of source locations (2925 source locations), or among all pairwise possibilities of the global grid. For each frequency, we counted the number of correlations that differed significantly between the blind and sighted (Wilcoxon rank-sum test, $p<0.05$, uncorrected). We treated differences with opposite signs separately. To assess the statistical significance of the number of altered correlations, we applied a random permutation procedure. We shuffled the participants' group affiliation (blind/sighted) randomly 50,000 times to estimate the number of positive and negative differences that occur by chance. This resulted in null hypothesis distributions that we denote by $D_{1}^{i}$, where $i$ indexes the different distributions for the effect directions (negative and positive differences) at all investigated frequencies (overall, 62 individual null hypothesis distributions). We corrected for multiple testing across all frequencies analyzed $(n=31$ frequency bins between 0.84 and $152.21 \mathrm{~Hz}$ ) as well as effect directions ( $n=2$, positive and negative) by comparing all effects to the maximum statistical resample distribution (Nichols and Holmes, 2002) using ranks: for each frequency and effect direction, we transformed the 50,000 resamples (obtained with the identical permutations) into a list of ranks $R_{\mathrm{r}}^{i}$. We then took the maximal rank across all individual $R_{1}^{i}$ values for each resample to construct a corresponding list of maximal ranks, denoted as $R_{1}^{\max }$. For each frequency and effect direction, this maximum rank distribution was then converted into maximum resample distributions, $D_{1 \text { max }}^{i}$. From these resample distributions, we derived corrected $p$ values for each frequency and effect direction. Computing the maximum resample distribution based on ranks rather than on the values themselves allows for combing distributions with different magnitudes and shapes as is the case for the different frequencies analyzed here. A maximum resample distribution of the raw values would be dominated by the individual distributions with the largest magnitudes, which severely affects the statistical sensitivity across frequencies.

The second step of the procedure was designed to localize brain regions that underlie the differences detected in the first step for the identified frequency ranges. For each brain-wide source location, we separately quantified the number of significantly altered correlations to visual cortex or to the rest of the brain (Wilcoxon rank-sum test, the blind individuals vs sighted control subjects, $p<0.05$, one-tailed test, uncorrected). For this analysis, we pooled correlation matrices of neighboring frequency bins that significantly differed between the groups as detected in the first step of the analysis. The selection of frequency ranges and effect directions introduces a statistical selection bias that needs to be corrected for (Kriegeskorte et al., 2009). To control for this bias and the number of statistical tests that we now performed at 2925 brain-wide source locations, we derived statistical thresholds by extending the rankbased procedure from the first step. The basic logic of the adjustment was to introduce the identical selection bias also to the resample distribution that is used to obtain $p$ value thresholds in this second step. To this end, we again shuffled the group affiliation of each participant 50,000 times using the identical permutations and derived all $R_{1}^{\max } D_{1}^{i}$ (see above). Additionally, we applied the same analysis for the two frequency ranges of interest by calculating resample distributions equivalent to $D_{1}^{j}$ as well as their corresponding rank lists $R_{1}$ based on the pooled correlation matrices and only for increases in the blind (Wilcoxon rank-sum test, the blind vs. sighted control subjects, $p<0.05$, one-tailed test, uncorrected). We denote these as $D_{\text {lavg }}^{j}$ and $R_{\text {lavg, }}^{j}$, respectively, where $j$ now indexes the two frequency ranges at the effect direction of interest. In addition, we counted for each resample the number of significantly altered power correlations with the visual sources or all other sources at each brainwide source location (Wilcoxon rank-sum test, the blind higher than sighted control subjects, $p<0.05$ one-tailed uncorrected). We retained only the maximum value across the entire brain, which resulted in maximum resample distributions, denoted as $D_{2 \max }^{j} D_{2}^{\max }$, that account for multiple testing across space. We then introduced the selection bias into the resampling distributions. For each resample $r$, we took the maximum rank occurring across all frequencies and effect directions from $R_{1 \max }$ and identified in $R_{\text {lavg }}^{j}$ ( $j$ being the index for the two selected frequency ranges) the resample $q$ that matched this maximum rank. We then selected from $D_{2 \max }^{j}$ the value at resample $q$ as the entry for resample $r$ in a new set of adjusted brain-wide resamples, denoted as $D_{\text {2adjusted. }}^{j}$. In other words, we made sure that each resample of the maximum statistic across the brain represented a value that had a rank equivalent to the maximally occurring rank across all frequencies and effect directions at that resample. This procedure incorporates the selection step in the resampling process and thus accounts for the statistical selection bias. From the adjusted resample distributions $D_{2 \text { adjusted }}^{j}$, we then derived corrected $p$ values for each cortical location and both selected frequency ranges.

Cross-frequency phase to amplitude coupling. For assessing crossfrequency coupling at a location $r$, we constructed a complex signal $C$ with the phase of a slow oscillation (frequency $f 1$ ) and the amplitude of a faster oscillation (frequency f2; Canolty et al., 2006), as follows:

$$
C_{r, f 1, f 2}(t)=\left|X_{r, f 2}(t)\right| \frac{X_{r, f 1}(t)}{\left|X_{r, f 1}(t)\right|} .
$$

Here $X_{r, f 1}$ and $X_{r, f 2}$ are the complex wavelet-transformed MEG data for the two frequencies. The raw coupling score was then calculated as the absolute of the average complex value across all $K$ time points, as follows:

$$
\mathrm{CSraw}_{r, f 1, f 2}=\left|\frac{\sum_{k}^{K} C_{r, f 1, f 2}(k)}{K}\right| .
$$

From this raw coupling score, we derived the normalized coupling score (CS) reported in all figures. We subtracted the mean and divided by the SD of a resampling distribution obtained by randomly shifting the phase to amplitude relation in time 1000 times and repeating the analysis, as follows:

$$
\mathrm{CS}_{r, f 1, f 2}=\left|\frac{\mathrm{CSraw}-\mu_{\text {shift }}}{\sigma_{\text {shift }}}\right| .
$$

The identical 1000 shift times were used in all participants. After normalization, the coupling scores were averaged across source locations. The cross-frequency coupling was computed for those source locations that exhibited increased correlations in both the delta and gamma range at a corrected significance level of $p<0.1(n=33)$. We computed the phaseamplitude coupling only for frequency pairs for which the amplitude frequency was at least twice the phase frequency.

To test whether the preferred phase of the cross-frequency coupling exhibited a concentration across participants, we used a procedure based on principal component analysis. Due to the noninvasive measurements and the nature of the source analysis that was used, the phase of neuronal oscillations is ambiguous with respect to a flip angle of $180^{\circ}$ (e.g., peak or trough). To account for this ambiguity, we used a procedure that tested for a consistent phase axis as opposed to a consistent phase angle.

In a first step, for each participant we computed the principal phase axis of the cross-frequency coupling. To this end, we selected the top 50\% of frequency pairs showing the strongest coupling scores within the two clusters of significant differences between the groups (see above). From these frequency pairs and all source locations, we only kept those for which the coupling score was $>0$ and obtained their phases, as follows:

$$
\text { CSphase }_{r, f 1, f 2}=\arg \left(\frac{\sum_{k}^{K} C_{r, f 1, f 2}(k)}{K}\right) .
$$

We then derived the principal phase axis as the principal component over the two-dimensional space defined by the cosine and sine of all CSphase $_{r, f 1, f 2}$ within a given subject. The principal phase axes are shown for each subject in the circle diagrams in Figure 3, $D$ and $E$.

In a second step, we analogously computed the group-level principal phase axis as the principal component of the subject-specific principal phase axes. To assess the significance of phase concentration, we compared the explained variance of the first principal component to the 

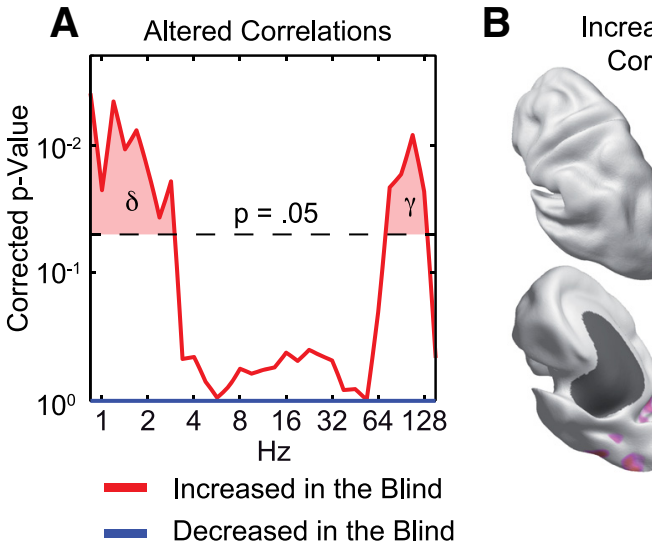

Correlations

$\delta$

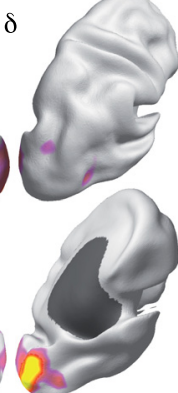

C Increased Gamma Correlations

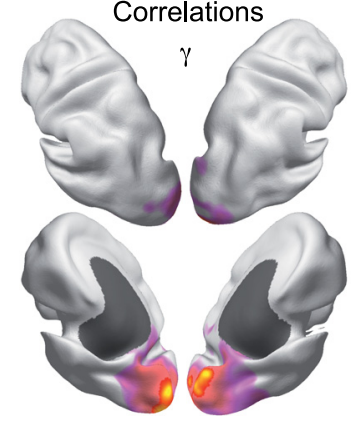

Corrected p-Value

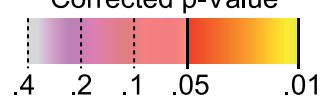

Figure 1. Visual cortex exhibits increased frequency-specific neuronal interactions in blind indviduals. $A, p$ value distribution of a permutation statistic assessing the number of altered power correlations between visual cortical areas (BA17 and BA18) and the rest of the brain across oscillation frequencies. Differences are analyzed separately for increased (red) and decreased (blue) correlations in blind compared with sighted individuals. $p$ values are corrected for the split into positive and negative differences, and all frequencies analyzed. $\boldsymbol{B}$, Cortical source locations that show increased delta $(\delta)$ correlations with the visual cortical areas. $p$ values are corrected for the frequency selection based on the data shown in $\boldsymbol{A}$ and for testing at all cortical source locations. $C$, Same as $\boldsymbol{B}$, but for increased gamma $(\gamma)$ correlations.

appropriate null hypothesis distribution constructed using random permutation. To this end, we randomly assigned the orientation of the subject-specific principal phase 1000 times.

The mean of the preferred phase across the blind group and its $95 \%$ confidence interval were derived by a bootstrapping procedure, computing the mean of the preferred phase across the group 1000 times while randomly drawing 11 datasets.

We constructed phase-sorted amplitude histograms by binning and averaging the amplitudes within 18 bins according to the phase of the slower oscillation with bin centers spanning the range from $-\pi$ to $\pi$ and a bin width of $20^{\circ}$. For each subject, before binning the amplitudes at a given source location and frequency pair, all phases within each voxel were rotated such that the subject's principal phase was aligned at zero.

Cluster-based permutation statistics. We used cluster-based permutation statistics in the analysis of cross-frequency coupling, power, and connectivity spectra. For all tests between groups, we used the Wilcoxon rank-sum test as the base statistic. Cluster sizes were determined as contiguous differences with $p<0.05$ (two-tailed test, uncorrected) and a consistent effect direction. We then repeated the analysis 1000 times, shuffling the participants randomly across the two groups and taking the maximum cluster size determined, as above. A cluster was determined to be significant at $p<0.05$ when its size exceeded the 95 th percentile of this maximum cluster size distribution. This procedure accounts for multiple testing (Nichols and Holmes, 2002). For assessing the within-group significance of cross-frequency coupling, we used Wilcoxon signed rank tests with $p<0.05$ (one tailed, uncorrected) to assess cluster sizes. To this end, we calculated the within-group statistic 1000 times based on the resamples obtained for each subject during the normalization procedure of the cross-frequency coupling strength. This resulted in a resample distribution of maximum cluster sizes within each of the two groups. A cluster was determined to be significant at $p<0.05$ within the groups when its size exceeded the 95th percentile of this maximum cluster size distribution.

\section{Results}

Visual areas of the blind exhibit an increase in frequencyspecific neuronal interactions

The high temporal resolution of MEG allows for resolving frequency-specific interactions of neuronal activity. In a first step, we analyzed how the power of frequency-specific neuronal activity cofluctuates across the brain of blind and sighted adults. We based this analysis on a recently introduced method for quantifying signal power correlations that is not confounded by the limited spatial resolution of noninvasive measurement tech- niques (Hipp et al., 2012). This method ensured that power correlations between any two locations were caused by genuinely distinct neuronal populations. We tested whether and, if so, in which frequency range, power correlations differed between blind and sighted subjects. Across a broad frequency range, we quantified the number of power correlations between visual cortical areas (source locations within BA17 and BA18) and the rest of the brain that differed between the congenitally blind and sighted (overall, 165,206 power correlations, Wilcoxon rank-sum tests, $p<0.05$ uncorrected). For each frequency, we then tested whether this number of differing power correlations was statistically significant using permutation statistics (Fig. 1A). Two frequency ranges exhibited significant increases of neuronal interactions in the blind. The delta frequency range was from $\sim 0.8$ to $2.8 \mathrm{~Hz}$ (permutation tests, $p<0.05$, corrected), and the gamma frequency range was from $\sim 76$ to $128 \mathrm{~Hz}$ (permutation tests, $p<0.05$, corrected). Thus, the signal power in visual cortex of the blind showed increased long-range correlations for slow and fast oscillatory activity.

These increased interactions specifically involved interactions within occipital cortical regions. For each source location throughout the brain and for both identified frequency ranges, we quantified the number of power correlations with the visual source locations increased in the blind (Wilcoxon rank-sum tests, $p<0.05$ ) and again used permutation statistics to assess their significance. In other words, we tested which brain regions showed increased power correlations with visual cortex in the blind compared with the sighted. We found significantly increased power correlations in occipital regions, covering caudal portions of the calcarine sulcus, the occipital pole, and posterior occipital cortex (Fig. $1 B, C$; permutation test, $p<0.05$, corrected). Even lowering the statistic threshold showed that the effect mainly was confined to visual cortex.

So far, the analyses were restricted to the brain-wide power correlations of the visual cortex. Thus, more widespread effects driven by interactions between regions outside visual cortex may have remained undetected. To test for such effects, we repeated the entire analysis, including all brain-wide power correlations (overall, 4,276,350 power correlations; Fig. 2). The results confirmed our above findings. Power correlations were increased in 
A

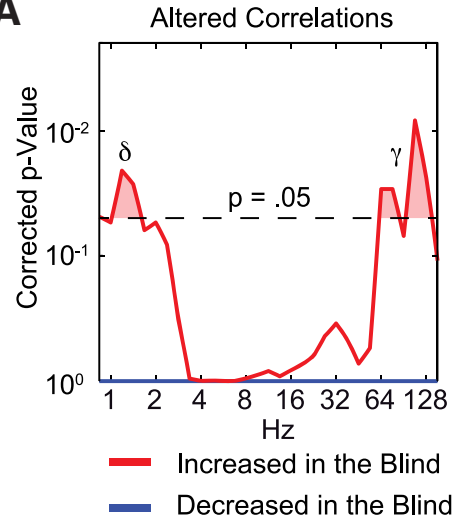

B

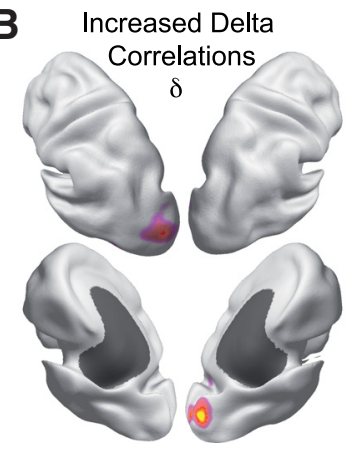

C Increased Gamma

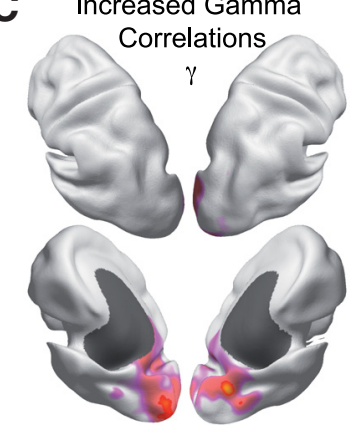

Corrected p-Value

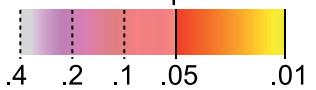

Figure 2. Global alterations of frequency-specific neuronal interactions in blind compared with sighted individuals. $A, p$ value distribution of a permutation statistic assessing the number of altered power-power correlations within all pairwise correlations among brain source locations across oscillation frequencies. Differences between the groups are analyzed separately for increased (red) and decreased (blue) correlations in blind compared with sighted individuals. The corrected $p$ value threshold is adjusted for the split into positive and negative differences, and all frequencies analyzed. $\boldsymbol{B}$, Cortical source locations that show increased brain-wide delta $(\delta)$ correlations. The $p$ values are adjusted for the selection made based on the data shown in $\boldsymbol{A}$ as well as the testing at all cortical source locations. $\boldsymbol{C}$, Same as $\boldsymbol{B}$ but for increased gamma $(\gamma)$ correlations.

the blind for visual cortical areas in the delta and gamma frequency range. We found no other significant differences in brainwide power correlations.

In sum, the blind's visual cortex spontaneously exhibited increased frequency-specific neuronal interactions in the delta and gamma frequency range.

\section{Delta phase modulates the gamma amplitude}

A growing body of evidence suggests that cortical computations rely on a nested organization of oscillatory processes in which the phase of slower oscillations modulates the amplitude of faster oscillations. Thus, in a next step, we investigated whether the two frequency ranges that showed increased interactions in blind visual cortex exhibited such cross-frequency phase-amplitude coupling. Indeed, we found significant cross-frequency coupling in the visual cortex of the blind and sighted. The congenitally blind group exhibited significant cross-frequency coupling, which was strongest for phases in the delta $(1.4-4 \mathrm{~Hz})$ frequency range extending also more weakly to theta $(4-8)$ and alpha $(8-$ 16) frequency phases. These low-frequency phases significantly predicted amplitudes in the gamma band (64-128 Hz; Fig. 3A; cluster-based permutation statistics, $p<0.05$ ). In contrast, the sighted showed significant cross-frequency coupling of theta to alpha $(5-13 \mathrm{~Hz})$ phases with amplitudes at lower gamma frequencies (19-76 Hz; Fig. 3B; cluster-based permutation statistics, $p<0.05)$. The direct comparison between the two groups revealed that the blind exhibited significantly stronger crossfrequency coupling between those frequencies that also showed enhanced power correlations. Cross-frequency coupling was significantly enhanced in the blind for delta to theta $(1.4-5 \mathrm{~Hz})$ and gamma $(76-152 \mathrm{~Hz})$ frequencies, while the sighted showed significantly stronger cross-frequency coupling for the alpha (5-16 $\mathrm{Hz}$ ) to lower gamma $(19-53 \mathrm{~Hz})$ frequencies (Fig. $3 C$; clusterbased permutation statistics, $p<0.05$ ). For the blind, we found a significant phase concentration of the delta-gamma coupling around the trough (or peak) of the phase oscillation (permutation test, $p=0.018$ ), while we did not find a significant phase concentration in the sighted group for the alpha-gamma coupling (permutation test, $p=0.5$; Fig. $3 D, E$ ). The noninvasive recordings together with the source analysis we used resulted in an ambiguity of the preferred phase of $\pi$ (see Materials and Meth- ods). The peak-to-trough amplitude modulations were $1.61 \%$ of the mean amplitude for the delta-gamma coupling in the blind, and $4.6 \%$ for the alpha-gamma coupling in the sighted (Fig. $3 D, E$ ).

In sum, we found in both groups that slower frequency phases modulated gamma frequency amplitudes. Furthermore, the visual cortex of blind individuals showed enhanced phase-amplitude coupling between those frequencies that also showed enhanced power correlations.

\section{Classical resting rhythms are reduced in the blind but show unchanged spatial correlation patterns}

A classical observation in the EEGs of blind individuals is a profound reduction of the posterior alpha $(10 \mathrm{~Hz})$ rhythm (Adrian and Matthews, 1934; Noebels et al., 1978; Kriegseis et al., 2006). In contrast, our above analyses show that brain-wide power correlations of visual cortex remain largely unaltered for these classical resting rhythms (Fig. $1 A ; 8-20 \mathrm{~Hz}$ ). To directly investigate the relation between signal strength and correlation, we thus quantified both average signal power and power correlations for early visual cortex (BA17; Fig. 4). The spectrum of the power correlation within early visual cortex exhibited a characteristic global peak in the alpha to beta $(8-20 \mathrm{~Hz})$ frequency range in both groups. Consistent with the above results (Figs. 1, 2), the blind exhibited additional peaks in the delta $(0.8-2 \mathrm{~Hz})$ and gamma $(76-152 \mathrm{~Hz})$ frequency range that were absent in the sighted (cluster-based permutation statistics, $p<0.05$ ). Interestingly, the power spectrum within early visual cortex of the blind showed differences compared with the sighted in a completely opposite manner. In both groups, the power spectrum also had a characteristic peak in the alpha to beta $(8-20 \mathrm{~Hz})$ frequency range. However, the signal power in the blind significantly differed from the sighted control subjects for alpha to beta frequencies (cluster-based permutation statistic, $p<0.05$ ), but not for delta or gamma frequencies. Thus, signal power and power correlations showed a double dissociation with opposite differences at distinct frequencies.

In a last step, we investigated whether differences in power correlations between the groups could be detected without using the signal orthogonalization. To this end, we again calculated the average power correlation for early visual cortex, but without using the orthogonalization technique. Interestingly, we did not find 
A

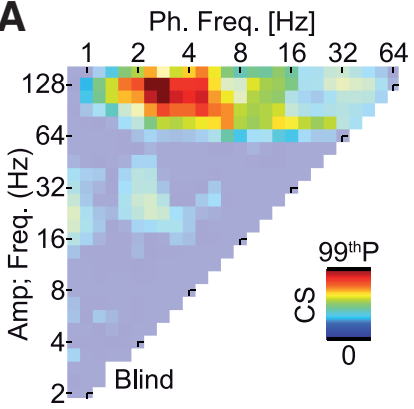

Blind: Delta-Gamma

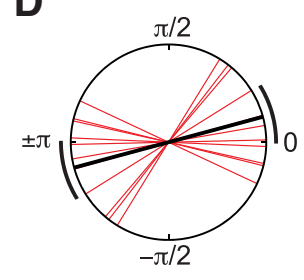

\section{C}

B Ph. Freq. [Hz]

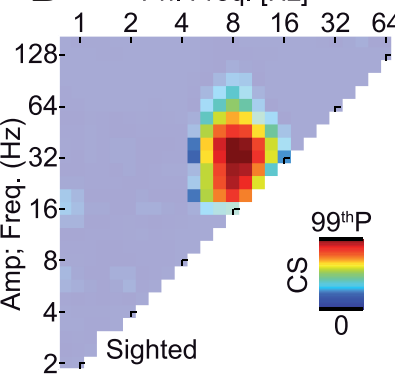

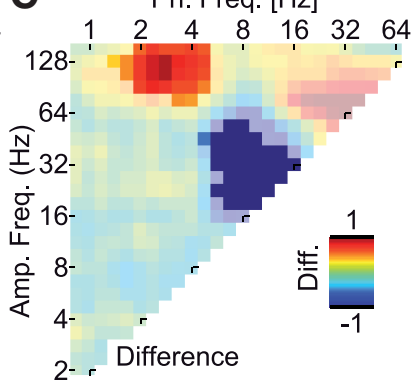

Sighted: Alpha-Gamma

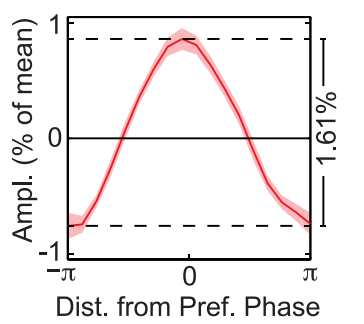

E
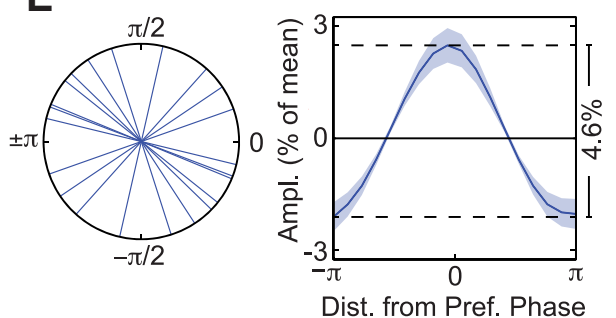

Figure 3. Cross-frequency phase-amplitude coupling in early visual areas dissociates between the blind and the sighted. $A$, Average cross-frequency CS for all phase and amplitude pairs for the blind. The color-saturated area shows a significant cluster of cross-frequency coupling, $p<0.05$. The color axis is scaled to the 99 th percentile of the coupling strength occurring across all frequency pairs, $c s_{99 p}=0.66$. B Same as $A$, but for sighted control subjects. $c s_{99 p}=2.14$. C, Difference in cross-frequency coupling strength between the groups for all phase and amplitude frequency pairs. The color-saturated areas show clusters with a significant difference between groups, $p<0.05$. D, Principal phase axes and phase-sorted amplitude histogram for the delta- gamma coupling found to be significantly stronger in the blind (see warm-colored cluster in $\boldsymbol{C}$ ). The black lines indicate the significant mean phase axis with its $95 \%$ confidence interval. The phase-sorted amplitude histogram shown as the mean across the group \pm SEM. $\boldsymbol{E}$, Same as $\boldsymbol{D}$, but for the sighted control subjects, and the alpha- gamma cross-frequency coupling was found to be significantly stronger in the sighted (see cold-colored cluster in C). No significant main-phase axis was found across the group.

any significant clusters of differences between the groups (clusterbased permutation statistic, $p>0.23$ ), suggesting that trivial signal correlations completely obscured the effect (Fig. 4C).

In sum, we found that classical resting rhythms $(8-20 \mathrm{~Hz})$ were reduced in the visual cortex of blind individuals, but exhibited a similar spatial correlation structure compared with sighted control subjects.

\section{Discussion}

Our results show altered interactions of delta $(1-4 \mathrm{~Hz})$ and gamma rhythms $(76-128 \mathrm{~Hz})$ as intrinsic characteristics of the visual cortex of blind individuals. In both frequency bands, interactions indexed by cofluctuations in signal power were enhanced within visual cortical areas. Furthermore, these oscillatory processes were functionally related in terms of cross-frequency phase-amplitude coupling. The specific frequency ranges involved and the types of interactions observed have important implications for the neuronal mechanisms that underlie the altered functional state of the visual cortex of blind individuals.

The gamma rhythm is a hallmark of active local processing in cortical circuits. Clocked by fast-spiking inhibitory interneurons (Hasenstaub et al., 2005; Cardin et al., 2009; Sohal et al., 2009), the gamma rhythm has been implicated in circuit mechanisms, such as gain control and signal amplification (Cardin et al., 2009; Sohal et al., 2009), and may contribute to basic sensory functions as well as higher cognitive processes (Singer, 1999; Tallon-Baudry and Bertrand, 1999; Fries et al., 2007; Jensen et al., 2007; Donner and Siegel, 2011). Gamma band responses in visual cortical areas are typically seen in noninvasive recordings during visual stimulation periods (Hoogenboom et al., 2006; Siegel et al., 2007; Hipp et al., 2011). Subsequent to the resting recordings that were analyzed here, the same congenitally blind participants took part in a study that involved auditory stimulation (Schepers et al., 2012).
Indeed, this study showed robust gamma band activity in visual cortex of the blind that was induced cross-modally by auditory stimulation and modulated by cognitive factors. Our present findings critically extend these results by showing that gamma band dynamics are present in the visual cortex of the blind even in the absence of stimulus drive or overt task demands. This finding may reflect a role of the visual cortex of blind individuals in nonvisual functions such as language and episodic memory (Amedi et al., 2003, 2004; Burton, 2003; Raz et al., 2005; Noppeney, 2007; Voss et al., 2010), which may account for a large fraction of introspective mentation during the resting condition and could underlie the increased metabolic demand of these regions in the blind (Wanet-Defalque et al., 1988; Veraart et al., 1990; De Volder et al., 1997). It should be noted that highfrequency activity $(>30 \mathrm{~Hz})$ does not necessarily reflect a single type of physiological process. Oscillatory and nonoscillatory processes may simultaneously contribute to the activity in this frequency range (Scheffer-Teixeira et al., 2013). Despite the band limited and peaked appearance of the gamma correlations found in the visual cortex of the blind (Fig. 4A), we cannot rule out that several different rhythmic and nonrhythmic components may contribute to the observed gamma band correlations.

Nonetheless, our findings suggest that the human cortical gamma band activity manifests in visual cortical areas even in the absence of appropriate sensory stimulation during postnatal development. The increased power correlations in gamma and delta activity across the visual cortex of blind individuals suggest that widely distributed neuronal populations largely share activity fluctuations. These increased lateral correlations are consistent with the lack of a differentiated cortical organization such as enlarged receptive fields and excessive horizontal connections due to disturbances in synaptic pruning and elimination of exuberant connectivity in early postnatal stages (Singer and Tretter, 
A Orthogonalized Correlation

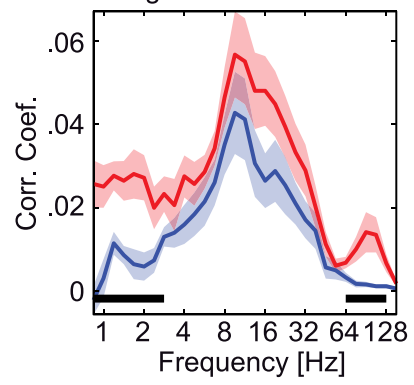

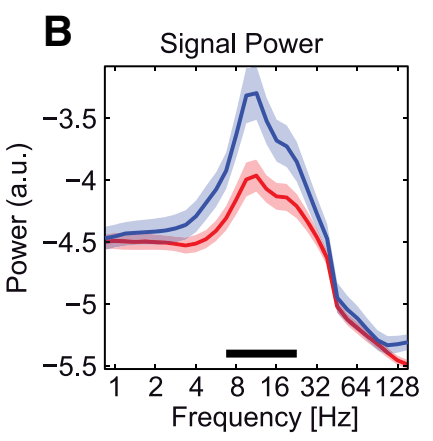

C Plain Correlation

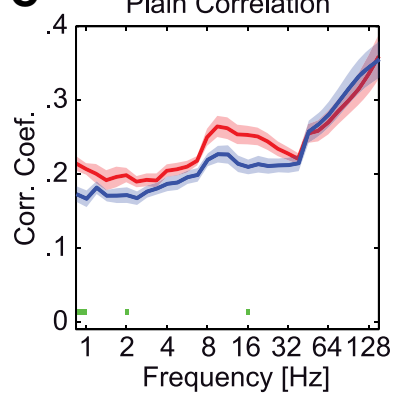

Blind

Diff. Cluster $p<.05$

Sighted

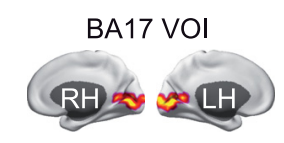

Figure 4. Local signal power shows a double dissociation from power correlations. $A$, Average orthogonalized power correlation between visual source locations within BA17 for the blind (red) and sighted (blue). Data are shown as the group mean \pm SEM. Significant clusters of differences between blind and sighted individuals across frequencies are marked as black lines, $p<0.05$. $\boldsymbol{B}$, Average power spectra for blind (red) and sighted (blue) individuals at visual source locations within BA17 for blind (red) and sighted (blue) individuals. Data are shown as the mean \pm SEM. Clusters with a significant difference between groups $(p<0.05)$ are shown as in $A$. C, Differences in power correlations were not detectable without signal orthogonalization. Average power correlation between source locations without signal orthogonalization for the blind (red) and sighted (blue). Data are shown as the mean \pm SEM. No significant cluster of differences was found. Individual tests that were significant at an uncorrected level are shown as green bars (Wilcoxon rank-sum test, $p<0.05$ ).

1976; Movshon and Van Sluyters, 1981; Huttenlocher et al., 1982; Callaway and Katz, 1991; Katz and Shatz, 1996). Indeed, a distinguishing feature of neuronal activity in visual cortex is the spread of fast-traveling waves along horizontal connections (Sato et al., 2012), which may underlie integration of information across large areas of cortex. Exuberant horizontal anatomical connectivity may be the substrate on which such waves of activity may lead to the increased lateral correlations of activity in the visual cortex of the blind demonstrated here.

Additional support for active functional processes intrinsically occurring across the visual cortex of blind individuals comes from the patterns of cross-frequency phase-amplitude coupling. We found that, in the blind, delta phases predicted gamma amplitude, with weaker coupling also extending to theta $(4-8 \mathrm{~Hz})$ and alpha $(8-16 \mathrm{~Hz})$ frequency phases. Such a pattern of crossfrequency coupling has been associated with normal cortical functioning and has been attributed to integrative processes across cortical laminae (Lakatos et al., 2005, 2008; Canolty et al., 2006; Osipova et al., 2008; Händel and Haarmeier, 2009; Schroeder and Lakatos, 2009; Voytek et al., 2010). Specifically in sensory cortical areas, phase-amplitude coupling of delta to theta phases and gamma amplitudes has been associated with increased spiking activity and the attentional routing of sensory signals (Lakatos et al., 2005, 2008; Schroeder and Lakatos, 2009; Whittingstall and Logothetis, 2009). The pattern of cross-frequency coupling found here is consistent with such processes spontaneously occurring in the visual cortex of blind individuals. In contrast, cross-frequency coupling between alpha $(10 \mathrm{~Hz})$ phases and gamma amplitudes, as found here in sighted individuals, has been shown to index rhythmic functional inhibition of cortical circuits, arising from the interplay of infragranular and supragranular neuronal populations (Spaak et al., 2012). Overall, the different patterns of cross-frequency coupling in blind and sighted individuals further support a divergence in functional state during silent wakefulness, with visual cortex of blind individuals exhibiting signatures of active functional processing and visual cortex of sighted individuals exhibiting signatures of functional inhibition.

The finding of reduced signal power for classical resting rhythms such as alpha frequency $(10 \mathrm{~Hz}$; Adrian and Matthews,
1934; Noebels et al., 1978; Kriegseis et al., 2006) in blind individuals further supports this conclusion. This reduction is consistent with a decrease of inhibitory processes (Singer and Tretter, 1976) indexed by alpha frequency activity (Klimesch et al., 2007; Osipova et al., 2008; Thut and Miniussi, 2009; Spaak et al., 2012) that may reflect reduced subcortical and, especially, thalamic anatomical connectivity (Singer and Tretter, 1976; Shimony et al., 2006). The reduction in alpha power also parallels the absence of inhibitory alpha-to-gamma cross-frequency coupling in blind individuals that we observed in sighted individuals. Interestingly, the pattern of decreased alpha-to-beta range power with a concurrent increase in gamma band activity matches the almost canonical pattern of frequency-specific neuronal responses in stimulation and task paradigms (Donner and Siegel, 2011). In sum, the pattern of local signal power changes also supports the view of visual cortex of blind individuals showing oscillatory signatures of increased excitability and functional processing.

All measures applied to oscillatory activity in our analysis (power correlations, cross-frequency phase-amplitude coupling, signal power) provided complementary information about the organization of neuronal activity in blind and sighted visual cortices. Specifically, power correlations provided a measure of interaction between spatially separate neuronal populations, while local cross-frequency coupling may index interactions across the cortical laminae. Knowledge about the patterns of activity that arise within the altered circuits of deprived sensory cortex are of highest relevance to approaches that aim to interface with and restore the function of lost sensory modalities (Merabet et al., 2005). Our results show that a quantification of multiple measures of oscillatory activity provides valuable detail about pathological brain organization. Altered power correlations were revealed by using a recent orthogonalization technique (Hipp et al., 2012) that removes spurious correlations, which otherwise masked the demonstrated effects. Our results indicate that, independent from mere signal strength, power correlations of orthogonalized signals provide a measure that is sensitive to pathological alterations of brain activity. This underlines the potential of frequency-specific power correlations for studying large-scale neuronal interactions, brain-computer interfaces, 
biofeedback, and other biomedical applications (Hipp et al., 2012; Siegel et al., 2012).

Together, the patterns of altered intrinsic neuronal activity that we found in blind individuals coherently suggest that their visual cortex is engaged in circuit mechanisms of active functional processing with oscillatory neuronal signatures similar to nondeprived active cortical circuits.

\section{References}

Adrian ED, Matthews BH (1934) The Berger rhythm: potential changes from the occipital lobes in man. Brain 57:355-385. CrossRef

Amedi A, Raz N, Pianka P, Malach R, Zohary E (2003) Early "visual” cortex activation correlates with superior verbal memory performance in the blind. Nat Neurosci 6:758-766. CrossRef Medline

Amedi A, Floel A, Knecht S, Zohary E, Cohen LG (2004) Transcranial magnetic stimulation of the occipital pole interferes with verbal processing in blind subjects. Nat Neurosci 7:1266-1270. CrossRef Medline

Bavelier D, Neville HJ (2002) Cross-modal plasticity: where and how? Nat Rev Neurosci 3:443-452. CrossRef Medline

Bedny M, Pascual-Leone A, Dodell-Feder D, Fedorenko E, Saxe R (2011) Language processing in the occipital cortex of congenitally blind adults. Proc Natl Acad Sci U S A 108:4429-4434. CrossRef Medline

Bragin A, Jandó G, Nádasdy Z, Hetke J, Wise K, Buzsáki G (1995) Gamma $(40-100 \mathrm{~Hz})$ oscillation in the hippocampus of the behaving rat. J Neurosci 15:47-60. Medline

Bridge H, Cowey A, Ragge N, Watkins K (2009) Imaging studies in congenital anophthalmia reveal preservation of brain architecture in "visual" cortex. Brain 132:3467-3480. CrossRef Medline

Brookes MJ, Woolrich MW, Barnes GR (2012) Measuring functional connectivity in MEG: a multivariate approach insensitive to linear source leakage. Neuroimage 63:910-920. CrossRef Medline

Bruns A, Eckhorn R, Jokeit H, Ebner A (2000) Amplitude envelope correlation detects coupling among incoherent brain signals. Neuroreport 11: 1509-1514. CrossRef Medline

Büchel C, Price C, Frackowiak RS, Friston K (1998) Different activation patterns in the visual cortex of late and congenitally blind subjects. Brain 121:409-419. CrossRef Medline

Burton H (2003) Visual cortex activity in early and late blind people. J Neurosci 23:4005-4011. Medline

Callaway EM, Katz LC (1991) Effects of binocular deprivation on the development of clustered horizontal connections in cat striate cortex. Proc Natl Acad Sci U S A 88:745-749. CrossRef Medline

Canolty RT, Knight RT (2010) The functional role of cross-frequency coupling. Trends Cogn Sci 14:506-515. CrossRef Medline

Canolty RT, Edwards E, Dalal SS, Soltani M, Nagarajan SS, Kirsch HE, Berger MS, Barbaro NM, Knight RT (2006) High gamma power is phaselocked to theta oscillations in human neocortex. Science 313:1626-1628. CrossRef Medline

Cardin JA, Carlén M, Meletis K, Knoblich U, Zhang F, Deisseroth K, Tsai LH, Moore CI (2009) Driving fast-spiking cells induces gamma rhythm and controls sensory responses. Nature 459:663-667. CrossRef Medline

Cohen LG, Celnik P, Pascual-Leone A, Corwell B, Falz L, Dambrosia J, Honda M, Sadato N, Gerloff C, Catalá MD, Hallett M (1997) Functional relevance of cross-modal plasticity in blind humans. Nature 389:180-183. CrossRef Medline

Collignon O, Voss P, Lassonde M, Lepore F (2009) Cross-modal plasticity for the spatial processing of sounds in visually deprived subjects. Exp Brain Res 192:343-358. CrossRef Medline

Collignon O, Vandewalle G, Voss P, Albouy G, Charbonneau G, Lassonde M, Lepore F (2011) Functional specialization for auditory-spatial processing in the occipital cortex of congenitally blind humans. Proc Natl Acad Sci U S A 108:4435-4440. CrossRef Medline

De Volder AG, Bol A, Blin J, Robert A, Arno P, Grandin C, Michel C, Veraart C (1997) Brain energy metabolism in early blind subjects: neural activity in the visual cortex. Brain Res 750:235-244. CrossRef Medline

Donner TH, Siegel M (2011) A framework for local cortical oscillation patterns. Trends Cogn Sci 15:191-199. CrossRef Medline

Fries P, Nikolić D, Singer W (2007) The gamma cycle. Trends Neurosci 30:309-316. CrossRef Medline

Gougoux F, Zatorre RJ, Lassonde M, Voss P, Lepore F (2005) A functional neuroimaging study of sound localization: visual cortex activity predicts performance in early-blind individuals. PLoS Biol 3:e27. CrossRef Medline

Gross J, Kujala J, Hamalainen M, Timmermann L, Schnitzler A, Salmelin R (2001) Dynamic imaging of coherent sources: studying neural interactions in the human brain. Proc Natl Acad Sci U S A 98:694-699. CrossRef Medline

Händel B, Haarmeier T (2009) Cross-frequency coupling of brain oscillations indicates the success in visual motion discrimination. Neuroimage 45:1040-1046. CrossRef Medline

Hasenstaub A, Shu Y, Haider B, Kraushaar U, Duque A, McCormick DA (2005) Inhibitory postsynaptic potentials carry synchronized frequency information in active cortical networks. Neuron 47:423-435. CrossRef Medline

Hipp JF, Engel AK, Siegel M (2011) Oscillatory synchronization in largescale cortical networks predicts perception. Neuron 69:387-396. CrossRef Medline

Hipp JF, Hawellek DJ, Corbetta M, Siegel M, Engel AK (2012) Large-scale cortical correlation structure of spontaneous oscillatory activity. Nat Neurosci 15:884-890. CrossRef Medline

Hoogenboom N, Schoffelen JM, Oostenveld R, Parkes LM, Fries P (2006) Localizing human visual gamma-band activity in frequency, time and space. Neuroimage 29:764-773. CrossRef Medline

Huttenlocher PR, de Courten C, Garey LJ, Van der Loos H (1982) Synaptogenesis in human visual-cortex-evidence for synapse elimination during normal development. Neurosci Lett 33:247-252. CrossRef Medline

Hyvärinen A (1999) Fast and robust fixed-point algorithms for independent component analysis. IEEE Trans Neural Netw 10:626-634. CrossRef Medline

Jensen O, Kaiser J, Lachaux JP (2007) Human gamma-frequency oscillations associated with attention and memory. Trends Neurosci 30:317324. CrossRef Medline

Jiang J, Zhu W, Shi F, Liu Y, Li J, Qin W, Li K, Yu C, Jiang T (2009) Thick visual cortex in the early blind. J Neurosci 29:2205-2211. CrossRef Medline

Katz LC, Shatz CJ (1996) Synaptic activity and the construction of cortical circuits. Science 274:1133-1138. CrossRef Medline

Klimesch W, Sauseng P, Hanslmayr S (2007) EEG alpha oscillations: the inhibition-timing hypothesis. Brain Res Rev 53:63-88. CrossRef Medline

Kriegeskorte N, Simmons WK, Bellgowan PS, Baker CI (2009) Circular analysis in systems neuroscience: the dangers of double dipping. Nat Neurosci 12:535-540. CrossRef Medline

Kriegseis A, Hennighausen E, Rösler F, Röder B (2006) Reduced EEG alpha activity over parieto-occipital brain areas in congenitally blind adults. Clin Neurophysiol 117:1560-1573. CrossRef Medline

Lakatos P, Shah AS, Knuth KH, Ulbert I, Karmos G, Schroeder CE (2005) An oscillatory hierarchy controlling neuronal excitability and stimulus processing in the auditory cortex. J Neurophysiol 94:1904-1911. CrossRef Medline

Lakatos P, Karmos G, Mehta AD, Ulbert I, Schroeder CE (2008) Entrainment of neuronal oscillations as a mechanism of attentional selection. Science 320:110-113. CrossRef Medline

Leopold DA, Murayama Y, Logothetis NK (2003) Very slow activity fluctuations in monkey visual cortex: implications for functional brain imaging. Cereb Cortex 13:422-433. CrossRef Medline

Merabet LB, Pascual-Leone A (2010) Neural reorganization following sensory loss: the opportunity of change. Nat Rev Neurosci 11:44-52. CrossRef Medline

Merabet LB, Rizzo JF, Amedi A, Somers DC, Pascual-Leone A (2005) What blindness can tell us about seeing again: merging neuroplasticity and neuroprostheses. Nat Rev Neurosci 6:71-77. CrossRef Medline

Movshon JA, Van Sluyters RC (1981) Visual neural development. Annu Rev Psychol 32:477-522. CrossRef Medline

Nichols TE, Holmes AP (2002) Nonparametric permutation tests for functional neuroimaging: a primer with examples. Hum Brain Mapp 15:1-25. CrossRef Medline

Noebels JL, Roth WT, Kopell BS (1978) Cortical slow potentials and the occipital EEG in congenital blindness. J Neurol Sci 37:51-58. CrossRef Medline

Nolte G (2003) The magnetic lead field theorem in the quasi-static approximation and its use for magnetoencephalography forward calculation in realistic volume conductors. Phys Med Biol 48:3637-3652. CrossRef Medline 
Noppeney U (2007) The effects of visual deprivation on functional and structural organization of the human brain. Neurosci Biobehav Rev 31 : 1169-1180. CrossRef Medline

Noppeney U, Friston KJ, Ashburner J, Frackowiak R, Price CJ (2005) Early visual deprivation induces structural plasticity in gray and white matter. Curr Biol 15:R488-R490. CrossRef Medline

Oostenveld R, Fries P, Maris E, Schoffelen JM (2011) FieldTrip: open source software for advanced analysis of MEG, EEG, and invasive electrophysiological data. Comput Intell Neurosci 2011:156869. CrossRef Medline

Osipova D, Hermes D, Jensen O (2008) Gamma power is phase-locked to posterior alpha activity. PLoS One 3:e3990. CrossRef Medline

Park HJ, Lee JD, Kim EY, Park B, Oh MK, Lee S, Kim JJ (2009) Morphological alterations in the congenital blind based on the analysis of cortical thickness and surface area. Neuroimage 47:98-106. CrossRef Medline

Raichle ME (2010) Two views of brain function. Trends Cogn Sci 14:180190. CrossRef Medline

Raz N, Amedi A, Zohary E (2005) V1 activation in congenitally blind humans is associated with episodic retrieval. Cereb Cortex 15:1459-1468. CrossRef Medline

Röder B, Teder-Sälejärvi W, Sterr A, Rösler F, Hillyard SA, Neville HJ (1999) Improved auditory spatial tuning in blind humans. Nature 400:162-166. CrossRef Medline

Röder B, Stock O, Bien S, Neville H, Rösler F (2002) Speech processing activates visual cortex in congenitally blind humans. Eur J Neurosci 16: 930-936. CrossRef Medline

Sadato N, Pascual-Leone A, Grafman J, Ibañez V, Deiber MP, Dold G, Hallett M (1996) Activation of the primary visual cortex by Braille reading in blind subjects. Nature 380:526-528. CrossRef Medline

Sato TK, Nauhaus I, Carandini M (2012) Traveling waves in visual cortex. Neuron 75:218-229. CrossRef Medline

Schack B, Vath N, Petsche H, Geissler HG, Möller E (2002) Phase-coupling of theta-gamma EEG rhythms during short-term memory processing. Int J Psychophysiol 44:143-163. CrossRef Medline

Scheffer-Teixeira R, Belchior H, Leão RN, Ribeiro S, Tort AB (2013) On high-frequency field oscillations $(>100 \mathrm{~Hz})$ and the spectral leakage of spiking activity. J Neurosci 33:1535-1539. CrossRef Medline

Schepers IM, Hipp JF, Schneider TR, Röder B, Engel AK (2012) Functionally specific oscillatory activity correlates between visual and auditory cortex in the blind. Brain 135:922-934. CrossRef Medline

Schroeder CE, Lakatos P (2009) Low-frequency neuronal oscillations as instruments of sensory selection. Trends Neurosci 32:9-18. CrossRef Medline

Shimony JS, Burton H, Epstein AA, McLaren DG, Sun SW, Snyder AZ (2006) Diffusion tensor imaging reveals white matter reorganization in early blind humans. Cereb Cortex 16:1653-1661. CrossRef Medline
Siegel M, Donner TH, Oostenveld R, Fries P, Engel AK (2007) Highfrequency activity in human visual cortex is modulated by visual motion strength. Cereb Cortex 17:732-741. CrossRef Medline

Siegel M, Donner TH, Engel AK (2012) Spectral fingerprints of large-scale neuronal interactions. Nat Rev Neurosci 13:121-134. CrossRef Medline

Singer W (1999) Neuronal synchrony: a versatile code for the definition of relations? Neuron 24:49-65, 111-125. CrossRef Medline

Singer W, Tretter F (1976) Receptive-field properties and neuronal connectivity in striate and parastriate cortex of contour-deprived cats. J Neurophysiol 39:613-630. Medline

Sohal VS, Zhang F, Yizhar O, Deisseroth K (2009) Parvalbumin neurons and gamma rhythms enhance cortical circuit performance. Nature 459: 698-702. CrossRef Medline

Spaak E, Bonnefond M, Maier A, Leopold DA, Jensen O (2012) Layerspecific entrainment of gamma-band neural activity by the alpha rhythm in monkey visual cortex. Curr Biol 22:2313-2318. CrossRef Medline

Tallon-Baudry C, Bertrand O (1999) Oscillatory gamma activity in humans and its role in object representation. Trends Cogn Sci 3:151-162. CrossRef Medline

Thut G, Miniussi C (2009) New insights into rhythmic brain activity from TMS-EEG studies. Trends Cogn Sci 13:182-189. CrossRef Medline

Van Essen DC (2005) A population-average, landmark- and surface-based (PALS) atlas of human cerebral cortex. Neuroimage 28:635-662. CrossRef Medline

Van Veen BD, van Drongelen W, Yuchtman M, Suzuki A (1997) Localization of brain electrical activity via linearly constrained minimum variance spatial filtering. IEEE Trans Biomed Eng 44:867-880. CrossRef Medline

Veraart C, De Volder AG, Wanet-Defalque MC, Bol A, Michel C, Goffinet AM (1990) Glucose utilization in human visual cortex is abnormally elevated in blindness of early onset but decreased in blindness of late onset. Brain Res 510:115-121. CrossRef Medline

Voss P, Collignon O, Lassonde M, Lepore F (2010) Adaptation to sensory loss. Cogn Sci 1:308-328. CrossRef

Voytek B, Canolty RT, Shestyuk A, Crone NE, Parvizi J, Knight RT (2010) Shifts in gamma phase-amplitude coupling frequency from theta to alpha over posterior cortex during visual tasks. Front Hum Neurosci 4:191. CrossRef Medline

Wanet-Defalque MC, Veraart C, De Volder A, Metz R, Michel C, Dooms G, Goffinet A (1988) High metabolic activity in the visual cortex of early blind human subjects. Brain Res 446:369-373. CrossRef Medline

Whittingstall K, Logothetis NK (2009) Frequency-band coupling in surface EEG reflects spiking activity in monkey visual cortex. Neuron 64:281-289. CrossRef Medline 